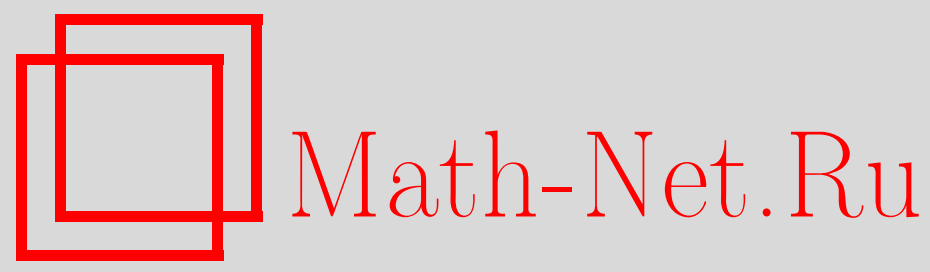

Я. Годуля, В. В. Старков, О граничном поведении в угле Штольца аналитических в круге функций, Матем. заметкu, 2002, том 71, выпуск 5, 652-661

DOI: https://doi.org/10.4213/mzm374

Использование Общероссийского математического портала Math-Net.Ru подразумевает, что вы прочитали и согласны с пользовательским соглашением http://www . mathnet.ru/rus/agreement

Параметры загрузки:

IP: 54.172 .240 .79

26 апреля 2023 г., 14:54:19

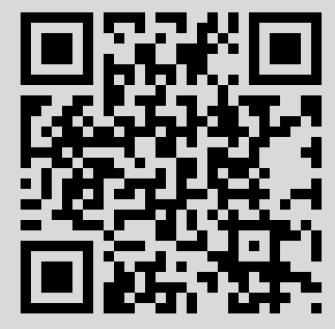




\title{
О ГРАНИЧНОМ ПОВЕДЕНИИ В УГЛЕ ШТОЛЬЦА АНАЛИТИЧЕСКИХ В КРУГЕ ФУНКЦИЙ
}

\author{
Я. Годуля, В.В. Старков
}

Заметка посвящена исследованию поведения в угле Штольца аналитических в круге функций. На этом пути, в частности, доказаны утверждения типа теоремы Харди-Литтлвуда, получены оценки на рост функции в угле Штольца.

Библиограффия: 3 названия.

Пусть $f$ - аналитическая в круге $\Delta=\{z:|z|<1\}$ функция. Эта статья посвящена решению некоторых задач о поведении $f(z)$ при $z \rightarrow e^{i \theta}(\theta \in \mathbb{R})$ в угле Штольца с вершиной $e^{i \theta}$, т.е. в области

$$
W_{\eta}=\left\{z \in \Delta:\left|\arg \left(1-z e^{-i \theta}\right)\right|<\eta,\left|1-z e^{-i \theta}\right|<\rho\right\},
$$

где $\eta \in(0, \pi / 2], 0<\rho<\cos \eta$. Важность такого рода задач обусловлена тем, что условие $W_{\eta} \ni z \rightarrow e^{i \theta}$ встречается во многих теоремах теории аналитических функций.

Интересно отметить, что техника доказательств полученных в этой статье результатов во многом взята нами из теории линейно-инвариантных семейств, введенных в [1] (см. [2]). Таким образом, методика, разработанная для специальных классов аналитических функций, какими являются линейно-инвариантные семейства, может успешно применяться и для исследования всего класса аналитических функций.

Tеорема 1. Пусть $f$ аналитична в $\Delta, c \in \mathbb{C} u \eta \in(0, \pi / 2]-$ фиксированные числа, $W_{\eta}-$ угол Штольиа с вершиной в $z=1$.

1) Если существует конечный предел

$$
\lim _{W_{\eta} \ni z \rightarrow 1}\left[f(z)(1-z)^{c}\right]=A,
$$

то для любого $\varepsilon \in(0, \eta)$ существует предел

$$
\lim _{W_{\eta-\varepsilon} \ni z \rightarrow 1}\left[f^{\prime}(z)(1-z)^{c+1}\right]=A c .
$$

2) Если для любого угла Штольиа $W_{\eta}$ существует конечный предел

$$
\lim _{W_{\eta} \ni z \rightarrow 1}\left[f(z)(1-z)^{c}\right]=A \neq 0,
$$

mo

$$
\lim _{a \rightarrow 1-} \frac{f((z+a) /(1+a z))}{f(a)}=\left(\frac{1+z}{1-z}\right)^{c}
$$

равномерно внутри $\Delta$.

3) Если функиия $f(z)(1-z)^{c}$ не ограничена в $W_{\eta}$ и $\operatorname{Re} c>0$, то функиия $f^{\prime}(z)(1-z)^{c+1}$ не ограничена в $W_{\eta}$. 
ЗАмЕчАниЕ 1. Здесь и далее, в тексте статьи при появлении многозначной аналитической функции считаем, что рассматривается главная ее ветвь: $1^{c}=1, \log 1=0$.

ЗАмЕчАниЕ 2. Простой пример: $f(z) \equiv 1, c<0$ показывает, что утверждение, обратное к утверждению 1$)$ теоремы 1 , неверно. Условие $\operatorname{Re} c>0$ в утверждении 3$)$ теоремы 1 является существенньг; об этом говорит, в частности, пример: $f(z)=\log (1-z)$, $c=0$.

ДоКАЗАТЕЛЬСТВо ТЕОРЕМЫ 1.1$)$ Любой круг $K$, лежащий в угле Штольца $W_{\eta}$ и касающийся его сторон, является образом круга $\{z:|z|<r(a)\}$ при отображении $w=(z+a) /(1+z a), a \in(-1,1)$. При этом центр $C$ круга $K$ и его радиус $R$ связаны с $r(a)$ формулами

$$
C=C(a)=\frac{a\left(1-r^{2}(a)\right)}{1-a^{2} r^{2}(a)}, \quad R=R(a)=\frac{\left(1-a^{2}\right) r(a)}{1-a^{2} r^{2}(a)}
$$

Поскольку $(1-C(a)) \sin \eta=R(a)$, то

$$
\left(1+a r^{2}(a)\right) \sin \eta=(1+a) r(a)
$$

Отсюда находим

$$
r(a)=\frac{a+1-\sqrt{(a+1)^{2}-4 a \sin ^{2} \eta}}{2 a \sin \eta} \underset{a \rightarrow 1-}{\rightarrow}=\operatorname{tg} \frac{\eta}{2}=r_{\eta}
$$

При малых $\delta>0$ круг $K_{\eta}(\delta)=\left\{z:|z| \leqslant r_{\eta}-\delta\right\} \subset\{z:|z|<r(a)\}$, если $a$ достаточно близко к 1. Отсюда и из утверждения 1) теоремы вытекает, что

$$
f\left(\frac{z+a}{1+a z}\right)\left(1-\frac{z+a}{1+a z}\right)^{c} \underset{a \rightarrow 1-}{\longrightarrow} A
$$

равномерно в круге $K_{\eta}(\delta)$. После дифференцирования (2) по $z$ получим

$$
\left[f^{\prime}\left(\frac{z+a}{1+a z}\right)\left(1-\frac{z+a}{1+a z}\right)^{c}-f\left(\frac{z+a}{1+a z}\right) c\left(1-\frac{z+a}{1+a z}\right)^{c-1}\right] \frac{1-a^{2}}{(1+a z)^{2}} \underset{a \rightarrow 1-}{\longrightarrow} 0
$$

что равносильно

$$
\left[f^{\prime}\left(\frac{z+a}{1+a z}\right)\left(1-\frac{z+a}{1+a z}\right)^{c+1}-f\left(\frac{z+a}{1+a z}\right) c\left(1-\frac{z+a}{1+a z}\right)^{c}\right] \frac{1+a}{(1+a z)(1-z)} \underset{a \rightarrow 1-}{\longrightarrow} 0
$$

равномерно в $K_{\eta}(\delta)$. Следовательно,

$$
f^{\prime}(w)(1-w)^{c+1}-f(w) c(1-w)^{c} \longrightarrow 0
$$

здесь $w \rightarrow 1$ в множестве $\Omega$, которое заполняют образы круга $K_{\eta}(\delta)$ при отображениях $w(z)=(z+a) /(1+a z), a \in(0,1)$. Вычисления, аналогичные проведенным вьше, 
показывают: если стороны угла $W_{\gamma}$ касаются образа круга $K_{\eta}(\delta)$ при его отображении функцией $w(z)=(z+a) /(1+a z)$, то

$$
\sin \gamma=\frac{\left(r_{\eta}-\delta\right)(1+a)}{1+a\left(r_{\eta}-\delta\right)^{2}} \underset{a \rightarrow 1-}{\longrightarrow} \frac{2\left(r_{\eta}-\delta\right)}{1+\left(r_{\eta}-\delta\right)^{2}}
$$

Сравнивая (4) и (1) и учитьвая, что $\delta$ сколь угодно мало, приходим к выводу, что для любого $\varepsilon \in(0, \eta)$ угол Штольца $W_{\eta-\varepsilon} \subset \Omega$, если $\delta$ достаточно близко к 0. Следовательно, (3) справедливо в любом угле Штольца $W_{\eta-\varepsilon}$, т.е.

$$
\lim _{W_{\eta-\varepsilon} \ni w \rightarrow 0}\left[f^{\prime}(w)(1-w)^{c+1}\right]=A c .
$$

2) Пусть для любого угла Штольца $W_{\eta}$ с вершиной в $z=1$

$$
\lim _{W_{\eta} \ni z \rightarrow 1}\left[f(z)(1-z)^{c}\right]=A \neq 0
$$

Тогда в любом угле Штольца $W_{\eta}$

$$
\lim _{W_{\eta} \ni z \rightarrow 1} \frac{f(z)}{g(z)}=A 2^{2-c}
$$

где $g(z)=(1+z)^{c-2} /(1-z)^{c}$. В частности, при $a \in(0,1)$

$$
\lim _{a \rightarrow 1-} \frac{f(a)}{g(a)}=A 2^{2-c} .
$$

Аналогично тому, как это сделано в при доказательстве утверждения 1), предельное соотношение (5) может быть записано в виде

$$
\lim _{a \rightarrow 1-} \frac{f((z+a) /(1+a z)) g(a)}{g((z+a) /(1+a z)) f(a)}=1
$$

причем сходимость здесь равномерная внутри $\Delta$, так как в круге $K_{\eta}(\delta)$ можно выбрать $\eta$ сколь угодно близким к $\pi / 2$, а $\delta$ - к нулю. Легко показать, что

$$
\frac{g((z+a) /(1+a z))}{g(a)(1+a z)^{2}} \equiv g(z)
$$

при $a \in(-1,1)$. Отсюда и из $(6)$ следует утверждение 2$)$ теоремы.

3) Пусть $\operatorname{Re} c>0$ и $f(z)(1-z)^{c}$ не ограничено в $W_{\eta}$. Предположим, что

$$
\sup _{z \in W_{\eta}}\left|f^{\prime}(z)(1-z)^{c+1}\right|<\infty
$$


т.е. существует постоянная $M>0$ такая, что $\left|f^{\prime}(z)\right| \leqslant M /|1-z|^{\operatorname{Re} c+1}$ для $z \in W_{\eta}$. Тогда для $z$ и $z_{0}$, лежащих на одном лучев $W_{\eta}$, исходящем из 1 , и таких, что $z_{0}$ фиксировано, а $z \rightarrow 1$ вдоль этого луча, в результате интегрирования по отрезку $\left[z_{0}, z\right]$ получим

$$
\begin{aligned}
\left|f\left(z_{0}\right)-f(z)\right| & \leqslant \int_{z}^{z_{0}}\left|f^{\prime}(s)\right||d s| \leqslant M \int_{z}^{z_{0}} \frac{|d(1-s)|}{|1-s|^{\operatorname{Re} c+1}} \\
& =M \int_{|1-z|}^{\left|1-z_{0}\right|} \frac{d t}{t^{\operatorname{Re} c+1}}=\frac{M}{\operatorname{Re} c}\left[\frac{1}{|1-z|^{\operatorname{Re} c}}-\frac{1}{\left|1-z_{0}\right|^{\operatorname{Re} c}}\right] .
\end{aligned}
$$

Следовательно,

$$
|f(z)||1-z|^{\operatorname{Re} c} \leqslant \frac{M}{\operatorname{Re} c}\left[1-\left|\frac{1-z}{1-z_{0}}\right|^{\operatorname{Re} c}\right]+\left|f\left(z_{0}\right)\right||1-z|^{\operatorname{Re} c} \underset{z \rightarrow 1}{\longrightarrow} \frac{M}{\operatorname{Re} c} .
$$

Отсюда вытекает ограниченность $\left|f(z)(1-z)^{c}\right|$ в $W_{\eta}$. Противоречие доказывает утверждение 3) теоремы 1.

Теорема 1 доказана.

Применяя $n$ раз утверждение 1 ) теоремы 1 , получаем

СледствИЕ 1. 1) В предположсениях утверждения 1) теоремы 1 в любом угле Штольца, меньшем $W_{\eta}$, существует предел

$$
\lim _{z \rightarrow 1}\left[f^{(n)}(z)(1-z)^{c+n}\right]=A c(c+1) \cdots(c+n-1)
$$

для любого $n \in \mathbb{N}$.

2) Если $f(z)(1-z)^{c}$ не ограничено в угле Штольца $W_{\eta} u \operatorname{Re} c>0$, то для любого $n \in \mathbb{N}$ функиия $f^{(n)}(z)(1-z)^{c+n}$ не ограничена в $W_{\eta}$.

СлЕДСТВИЕ 2. Если $f$ регулярна в $\Delta, c \in \mathbb{C}, \theta \in \mathbb{R}, W_{\eta}-$ угол Штольиа с верииной в $e^{i \theta}$ и существует конечный предел

$$
\lim _{W_{\eta} \ni z \rightarrow e^{i \theta}}\left[f(z)\left(1-z e^{-i \theta}\right)^{c}\right]=A,
$$

то в любом меньшем угле Штольиа с верииной в $z=e^{i \eta}$ существует предел

$$
\lim _{z \rightarrow e^{i \theta}}\left[f^{\prime}(z)\left(1-z e^{-i \theta}\right)^{c+1}\right]=A c e^{-i \theta} .
$$

Вешественным аналогом теоремы 1 можно считать теорему Харди и Литтльвуда [3, c. 215-216], а именно

если функиия $f(x)$ дифференцируема на $(0,1), f^{\prime}(x)$ возрастает на $(0,1), c>0 u$

$$
\lim _{x \rightarrow 1-} f(x)(1-x)^{c}=A>0,
$$

то существует предел

$$
\lim _{x \rightarrow 1-} f^{\prime}(x)(1-x)^{c+1}=A c .
$$

Из доказательства утверждения 1) теоремы 1 вытекает справедливость следующего утверждения 
МОДИФИКАЦИЯ ТЕОРЕМЫ ХАРДИ-ЛИТТЛЬВУДА. Если функиия $f(x)$ определена на интервале $\left(r_{0}, 1\right), r_{0} \in(0,1)$, и является сужением на этот интервал функиии $f(z)$, аналитической в некотором угле Штольиа $W_{\eta}$ с вершиной в $z=1, u n р и$ фиксированном $с \in \mathbb{C}$ существует предел

$$
\lim _{W_{\eta} \ni z \rightarrow 1}\left[f(z)(1-z)^{c}\right]=A \in \mathbb{C},
$$

то существует предел

$$
\lim _{x \rightarrow 1-}\left[f^{\prime}(x)(1-x)^{c+1}\right]=A c .
$$

Если при этом функция $f$ является нормальной в $\Delta$, то вместо существования углового предела в (7) достаточно потребовать существования радиального предела

$$
\lim _{x \rightarrow 1-}\left[f(x)(1-x)^{c}\right]=A \in \mathbb{C} .
$$

Аналогично тому, как доказано утверждение 1 ) теоремы 1 , можно доказать, например, следуюшее утверждение.

Теорема 2. Пусть $f$ аналитична в $\Delta, c, \mu \in \mathbb{C}$. Если (обозначения из теоремы 1) существует конечный предел

$$
\lim _{W_{\eta} \ni z \rightarrow 1}\left[f(z)(1-z)^{c}\left(\log \frac{1}{1-z}\right)^{\mu}\right]=A \quad\left(u \Omega u \lim _{W_{\eta} \ni z \rightarrow 1}\left[f(z) \exp \frac{c}{1-z}\right]=A\right),
$$

mo

$$
\begin{aligned}
& \lim _{W_{\eta-\varepsilon} \ni z \rightarrow 1}\left[f^{\prime}(z)(1-z)^{c+1}\left(\log \frac{1}{1-z}\right)^{\mu}\right]=c A \\
& \left(\text { uлu } \lim _{W_{\eta-\varepsilon} \ni z \rightarrow 1}\left[f^{\prime}(z)(1-z)^{2} \exp \frac{c}{1-z}\right]=-c A\right) \text {. }
\end{aligned}
$$

ОПРЕДЕЛЕниЕ 1. Пусть $f$ аналитична в $\Delta$. Точку $e^{i \theta} \in \partial \Delta$ назовем точкой роста $\oint y н к u и и ~ f$, если существует угол Штольца $W_{\eta}$ с вершиной в $e^{i \theta}$ и $\beta>0$ такие, что

$$
\limsup _{W_{\eta} \ni z \rightarrow e^{i \theta}}\left[|f(z)|(1-|z|)^{\beta}\right]>0 .
$$

Супремум таких $\beta$ обозначим $\delta_{\eta}=\delta_{\eta}(f) \in(0, \infty]$ и назовем порядком роста функиии $f$ в угле $W_{\eta}$, а число $\delta=\delta(f)=\sup _{\eta \in(0, \pi / 2)} \delta_{\eta}-$ порядком роста функиии $f$ в mочке $e^{i \theta}$.

Порядок роста $\delta_{\eta}$ данной функции $f$ зависит, вообще говоря, от $\eta$ (см. теорему 3 ) и является неубывающей по $\eta$ функцией. Из определения 1 следует, что для любого $\varepsilon>0$ существует постоянная $M_{\varepsilon}>0$ такая, что $|f(z)| \leqslant M_{\varepsilon} /(1-|z|)^{\delta_{\eta}+\varepsilon}$ для $z \in W_{\eta}$. Связь между порядками роста в угле функций $f$ и $f^{\prime}$ описывает следующая теорема. 
Tеорема 3. Если е $e^{i \theta}$ - точка роста функиии $f$, то е $e^{i \theta}$ является точкой росma $f^{\prime}$, при этом $\delta_{\eta}\left(f^{\prime}\right) \geqslant \delta(f)+1 ;$ однако для любого $\varepsilon \in(0, \eta) \delta_{\eta-\varepsilon}\left(f^{\prime}\right) \leqslant \delta_{\eta}(f)+1$. Если $\delta_{\eta}(f)<\infty$, то для любого $\alpha \in\left(\delta_{\eta}(f)+1, \delta_{\eta}(f)+1+\pi / 2 \eta\right]$ существует функиия $f$ с точкой роста в $e^{i \theta}$ такая, что $\delta_{\eta}\left(f^{\prime}\right)=\alpha$.

ДоКАЗАТЕЛЬСТво. Если $0<\beta<\delta_{\eta}$, то

$$
\limsup _{W_{\eta} \ni z \rightarrow e^{i \theta}}\left[|f(z)|(1-|z|)^{\beta}\right]=\infty .
$$

Покажем, что и

$$
\limsup _{W_{\eta} \ni z \rightarrow e^{i \theta}}\left[\left|f^{\prime}(z)\right|(1-|z|)^{\beta+1}\right]=\infty .
$$

Пусть это не так. Тогда существует постоянная $M>0$ такая, что $\left|f^{\prime}(z)\right| \leqslant M /(1-|z|)^{\beta+1}$ для всех $z \in W_{\eta}$, достаточно близких к $e^{i \theta}$. Возьмем такие $z, z_{0} \in W_{\eta}$, достаточно близкие к $e^{i \theta}$, фиксируем $z_{0}, z$ устремим к $e^{i \theta}$ в $W_{\eta}$. Интегрируя по отрезку $\left[z_{0}, z\right]$, оценим разность

$$
\begin{aligned}
\left|f(z)-f\left(z_{0}\right)\right| & =\left|\int_{z_{0}}^{z} f^{\prime}(s) d s\right| \leqslant M \int_{z_{0}}^{z} \frac{|d s|}{(1-|s|)^{\beta+1}} \\
& \leqslant \frac{M}{2 \cos \eta} \int_{z_{0}}^{z} \frac{d|s|}{(1-|s|)^{\beta+1}}=\frac{M}{2 \beta \cos \eta}\left[\frac{1}{(1-|z|)^{\beta}}-\frac{1}{\left(1-\left|z_{0}\right|\right)^{\beta}}\right] .
\end{aligned}
$$

Следовательно,

$$
|f(z)|(1-|z|)^{\beta} \leqslant\left|f\left(z_{0}\right)\right|(1-|z|)^{\beta}+\frac{M}{2 \beta \cos \eta}\left[1-\left(\frac{1-|z|}{1-\left|z_{0}\right|}\right)^{\beta}\right] \leqslant \frac{M}{\beta \cos \eta},
$$

если $z$ достаточно близко к $e^{i \theta}$. Следовательно, $|f(z)|(1-|z|)^{\beta}$ ограничено при $z \in W_{\eta}$, $z \rightarrow e^{i \theta}$. Противоречие показьвает, что $e^{i \theta}$ - точка роста $f^{\prime}$, причем порядок роста $f^{\prime}$ в угле $W_{\eta}$ не меньше $\delta_{\eta}+1$.

Если $\delta_{\eta}<\infty$ и $\beta>\delta_{\eta}$, то

$$
\limsup _{W_{\eta} \ni z \rightarrow e^{i \theta}}\left[|f(z)|(1-|z|)^{\beta}\right]=0 \Longrightarrow \lim _{W_{\eta} \ni z \rightarrow e^{i \theta}}\left[\left|f(z)(1-z)^{\beta}\right|\right]=0 .
$$

Применяя утверждение 1) теоремы 1 при $A=0$, получим

$$
\lim _{W_{\eta-\varepsilon} \ni z \rightarrow e^{i \theta}}\left[\left|f^{\prime}(z)(1-z)^{\beta+1}\right|\right]=0, \quad \varepsilon \in(0,1) .
$$

Следовательно,

$$
\lim _{W_{\eta-\varepsilon} \ni z \rightarrow e^{i \theta}}\left[\left|f^{\prime}(z)\right|(1-|z|)^{\beta+1}\right]=0,
$$

т.е. порядок роста $f^{\prime}$ в угле $W_{\eta-\varepsilon}$ не превосходит $\delta_{\eta}+1$.

Теперь фиксируем $\alpha \in(0, \pi / 2 \eta]$. Функция $\zeta=1 /\left(1-z e^{-i \theta}\right)^{\alpha}$ переводит $W_{\eta}$ однолистно в область $\{\zeta:|\arg \zeta|<\eta \alpha\} \backslash \Omega$, где $\Omega$ - ограниченное множество. Поэтому функция $t(z)=i e^{i \eta \alpha} /\left(1-z e^{-i \theta}\right)^{\alpha}$ однолистно отображает $W_{\eta}$ в область, лежащую в 
левой полуплоскости и содержащую луч $\left\{i \tau-1: \tau \geqslant \tau_{0}\right\}=l$. Обозначим $\Gamma=t^{-1}(l)$. Если $l \ni t \rightarrow \infty$, то соответствующая точка $z \in \Gamma$ стремится к $e^{i \theta}$. Поскольку $\Gamma \subset W_{\eta}$, то для функции

$$
f(z)=\frac{\exp \left[i e^{i \eta \alpha}\left(1-z e^{-i \theta}\right)^{-\alpha}\right]}{\left(1-z e^{-i \theta}\right)^{\delta}}, \quad \delta>0
$$

имеем

$$
\limsup _{\Gamma \ni z \rightarrow 1}\left[|f(z)|(1-|z|)^{\delta}\right]=e^{-1} \limsup _{\Gamma \ni z \rightarrow 1}\left(\frac{1-|z|}{\left|1-z e^{-i \theta}\right|}\right)^{\delta}>0 .
$$

С другой стороны,

$$
\limsup _{W_{\eta} \ni z \rightarrow 1}\left[|f(z)|(1-|z|)^{\delta}\right] \leqslant \limsup _{W_{\eta} \ni z \rightarrow 1}\left(\frac{1-|z|}{\mid 1-z e^{-i \theta \mid}}\right)^{\delta} \leqslant 1 .
$$

Следовательно, порядок роста $f$ в угле $W_{\eta}$ равен $\delta$. Однако порядок роста $f^{\prime}$ в угле $W_{\eta}$ равен $\delta+1+\alpha$, так как

$$
f^{\prime}(z)=f(z)\left[\frac{\delta}{e^{i \theta}-z}+\frac{\alpha i e^{i(\eta \alpha-\theta)}}{\left(1-z e^{-i \theta}\right)^{\alpha+1}}\right]
$$

Теорема 3 доказана.

Таким образом, если величина угла $W_{\eta}$ стремится к нулю, то $\delta_{\eta}\left(f^{\prime}\right)-\delta_{\eta}(f)$ может стремиться к $\infty$.

При конформных автоморфизмах $w(z)=(z+a) /(1+\bar{a} z), a \in \Delta$, единичного круга точки роста функции $f(z, a)=f((z+a) /(1+\bar{a} z))$ переходят в точки роста функции $f$.

Теорема 4. Пусть функиия $f$ аналитична в $\Delta, a \in \Delta$. Чтобы функция $f(z, a)$ имела в точке $e^{i \phi}$ порядок роста $\delta$, необходимо и достаточно, чтобы порядок роста $f$ в точке $e^{i \gamma}=\left(e^{i \phi}+a\right) /\left(1+\bar{a} e^{i \phi}\right)$ бил равен $\delta$.

ДокАЗАтЕльСтво. Если функция $f(z, a)$ имеет в точке $e^{i \phi}$ порядок роста $\delta$, то для любого $\varepsilon>0$ существует $\eta \in(0, \pi / 2)$ такое, что $0 \leqslant \delta-\delta_{\eta}(f(z, a))<\varepsilon$, где

$$
\delta_{\eta}(f(z, a))=\sup \left\{\beta>0: \limsup _{W_{\eta} \ni z \rightarrow e^{i \phi}}\left[|f(z, a)|(1-|z|)^{\beta}\right]>0\right\} .
$$

Если $W_{\eta} \ni z \rightarrow e^{i \phi}$, то

$$
w=\frac{z+a}{1+\bar{a} z} \rightarrow \frac{e^{i \phi}+a}{1+\bar{a} e^{i \phi}}=e^{i \gamma}
$$

причем можно считать, что $w \in W_{\eta+\lambda}$, где $\lambda>0$ и сколь угодно близко к 0 . Поскольку для $z$, близких к $e^{i \phi}, z \in W_{\eta}$,

$$
\frac{1-|z|}{1-|w(z)|} \geqslant \frac{\left|e^{i \phi}-z\right|}{\left|e^{i \gamma}-w(z)\right|} \frac{\cos \eta}{2}=\frac{\cos \eta}{2} \frac{\left|\left(1+\bar{a} e^{i \phi}\right)(1+\bar{a} z)\right|}{1-|a|^{2}} \rightarrow \frac{\cos \eta}{2} \frac{\left|1+\bar{a} e^{i \phi}\right|^{2}}{1-|a|^{2}}>0
$$


то для каждого $\beta>0$, удовлетворяющего неравенству (8),

$$
\limsup _{W_{\eta+\lambda} \ni w \rightarrow e^{i \gamma}}\left[|f(w)|(1-|w|)^{\beta}\right]>0
$$

Поэтому $e^{i \gamma}$ - точка роста функции $f(z)$ и $\delta_{\eta+\lambda}(f(z)) \geqslant \delta_{\eta}(f(z, a))>\delta-\varepsilon$. Следовательно, порядок роста функции $f(z)$ в точке $e^{i \gamma}$ не меньше $\delta-\varepsilon$, значит он не меньше $\delta$ (это следует из произвольности $\varepsilon>0$ ).

Если теперь в предыдущих рассуждениях взять функцию $f(z, a)$ вместо $f(z)$ и вместо $w(z)$ автоморфизм $(z-a) /(1-\bar{a} z)$, то получим утверждение теоремы.

Теорема 4 доказана.

Теорема 5. Пусть $e^{i \theta}-$ точка роста функиии $f$. Если для некоторого угла Штольиа $W_{\eta}$ с верииной в $e^{i \theta}$ и для некоторого фиксированного $c \in \mathbb{C}$

$$
\begin{aligned}
0 & <\delta^{\prime}=\liminf _{z=r e^{i \theta} \rightarrow e^{i \theta}} \operatorname{Re}\left\{\frac{f^{\prime}(z)}{f(z)-c}\left(e^{i \theta}-z\right)\right\} \\
& \leqslant \limsup _{W_{\eta} \ni z \rightarrow e^{i \theta}} \operatorname{Re}\left\{\frac{f^{\prime}(z)}{f(z)-c}\left(e^{i \theta}-z\right)\right\}=\delta^{\prime \prime}<+\infty
\end{aligned}
$$

әде $r \in(0,1)$, то порядок роста функиии $f$ в угле $W_{\eta}$ удовлетворяет неравенству $\delta^{\prime} \leqslant \delta_{\eta}(f) \leqslant \delta^{\prime \prime} ;$ причем нижняя оченка справедлива для любого $\eta \in(0, \pi / 2)$. Если же определенное выше $\delta^{\prime \prime} \leqslant 0$ для любого угла Штольца $W_{\eta}$ с верииной в $e^{i \theta}$, то $e^{i \theta}$ не является точкой роста $f$.

ДокАЗАТЕЛЬСтво. Из определения $\delta^{\prime}$ вытекает, что для любого $\varepsilon>0$ существует $r_{\varepsilon} \in(0,1)$ такое, что для любого $r \in\left[r_{\varepsilon}, 1\right)$

$$
\operatorname{Re}\left\{\frac{f^{\prime}\left(r e^{i \theta}\right)}{f\left(r e^{i \theta}\right)-c} e^{i \theta}\right\}>\frac{\delta^{\prime}-\varepsilon}{1-r} .
$$

Следовательно, функция $\operatorname{Re}\left\{\log \left[f\left(r e^{i \theta}\right)-c\right]\right\}+\left(\delta^{\prime}-\varepsilon\right) \log (1-r)$ возрастает на интервале $\left(r_{\varepsilon}, 1\right)$. Поэтому существует предел

$$
\lim _{r \rightarrow 1}\left[\left|f\left(r e^{i \theta}\right)-c\right|(1-r)^{\delta^{\prime}-\varepsilon}\right]>0
$$

Следовательно, существует

$$
\lim _{r \rightarrow 1}\left[\left|f\left(r e^{i \theta}\right)\right|(1-r)^{\delta^{\prime}-\varepsilon}\right]>0
$$

В силу произвольности $\varepsilon>0$ отсюда получаем неравенство $\delta^{\prime} \leqslant \delta_{\eta}(f)$ в любом угле Штольца $W_{\eta}$ с вершиной в $e^{i \theta}$.

Из определения $\delta^{\prime \prime}$ следует, что

$$
\operatorname{Re}\left\{\frac{f^{\prime}(z)}{f(z)-c}\left(e^{i \theta}-z\right)\right\}<\delta^{\prime \prime}+\varepsilon
$$


при $z \in W_{\eta},|z|>r_{\varepsilon} \in(0,1)$. Обозначим $L$ пересечение угла $W_{\eta}$ с лучом $\left\{z: \arg \left(e^{i \theta}-z\right)\right.$ $=\varphi\}$ ( $\varphi$ фиксировано), лежащим в $W_{\eta}$. На этом луче фиксируем $z_{0},\left|z_{0}\right|=r_{\varepsilon}$, и пусть $z \in\left[z_{0}, e^{i \theta}\right)$. Обозначим

$$
\lambda=\left|\frac{e^{i \theta}-z}{e^{i \theta}-z_{0}}\right|=\frac{e^{i \theta}-z}{e^{i \theta}-z_{0}} \in(0,1]
$$

Тогда $z=e^{i \theta}-\lambda\left(e^{i \theta}-z_{0}\right)$ и (10) примет вид

$$
\operatorname{Re}\left\{\frac{f^{\prime}\left(e^{i \theta}-\lambda\left(e^{i \theta}-z_{0}\right)\right)}{f\left(e^{i \theta}-\lambda\left(e^{i \theta}-z_{0}\right)\right)-c}\left(e^{i \theta}-z_{0}\right)\right\} \leqslant \frac{\delta^{\prime \prime}+\varepsilon}{\lambda} .
$$

Интегрируя это неравенство по отрезку $[\lambda, 1]$, получим

$$
\begin{aligned}
\log |f(z)-c|-\log \left|f\left(z_{0}\right)-c\right| & \leqslant\left(\delta^{\prime \prime}+\varepsilon\right) \log \left|\frac{e^{i \theta}-z_{0}}{e^{i \theta}-z}\right| \\
\Longleftrightarrow|f(z)-c|\left|e^{i \theta}-z\right|^{\delta^{\prime \prime}+\varepsilon} & \leqslant\left|f\left(z_{0}\right)-c\right|\left|e^{i \theta}-z\right|^{\delta^{\prime \prime}+\varepsilon} \\
& \leqslant \max _{z \in W_{\eta},|z|=r_{\varepsilon}}\left[|f(z)-c|\left|e^{i \theta}-z\right|^{\delta^{\prime \prime}+\varepsilon}\right] .
\end{aligned}
$$

Следовательно, величина $|f(z)-c|(1-|z|)^{\delta^{\prime \prime}+\varepsilon}$ ограничена в $W_{\eta}$. Поэтому $|f(z)| \times$ $(1-|z|)^{\delta^{\prime \prime}+\varepsilon}$ ограничена в $W_{\eta}$, так как $\delta^{\prime \prime}>0$. В силу произвольности $\varepsilon$ отсюда получаем неравенство $\delta_{\eta}(f) \leqslant \delta^{\prime \prime}$.

Если в любом угле Штольца $\delta^{\prime \prime} \leqslant 0$, то в приведенных выше рассуждениях для фиксированного угла $W_{\eta}$ достаточно положить $\delta^{\prime \prime}=0$. Тогда получим неравенство

$$
|f(z)|(1-|z|)^{\varepsilon} \leqslant M_{\varepsilon}(\eta)=\mathrm{const}, \quad z \in W_{\eta} .
$$

Поскольку неравенство справедливо для любого $\varepsilon>0$, то $e^{i \theta}$ не является точкой роста $f$.

Теорема 5 доказана.

Универсальное линейно-инвариантное семейство $\mathscr{U}_{\alpha}(\alpha \geqslant 1)$, введенное Поммеренке [1], представляет собой множество всех локально однолистньх в $\Delta$ функций $f(z)=z+\cdots$, для которых

$$
\operatorname{ord} f=\sup _{z \in \Delta}\left|\frac{f^{\prime \prime}(z)}{f^{\prime}(z)} \frac{1-|z|^{2}}{2}-\bar{z}\right|=\sup _{z \in \Delta}|t(z, f)| \leqslant \alpha
$$

здесь

$$
t(z, f)=\frac{z f^{\prime \prime}(z)}{f^{\prime}(z)} \frac{1-|z|^{2}}{2|z|}-|z| .
$$

Для таких функций теорему 5 можно сформулировать в терминах $t(z, f)$. 
Tеорема 5'. Пусть $f$ аналитична и локально однолистна в $\Delta, e^{i \theta}-$ точка роста $f^{\prime}$. Если в некотором угле Штольиа $W_{\eta}$ выполняется неравенство

$$
-1<\delta_{1}=\liminf _{z=r e^{i \theta} \rightarrow e^{i \theta}} \operatorname{Re} t(z, f) \leqslant \limsup _{W_{\eta} \ni z \rightarrow e^{i \theta}} \operatorname{Re} t(z, f)=\delta_{2}<+\infty,
$$

где $r \in(0,1)$, то $\delta_{1}+1 \leqslant \delta_{\eta}\left(f^{\prime}\right) \leqslant \delta_{2}+1$, причем левое неравенство справедливо для любого $\eta \in(0, \pi / 2)$. Если $\delta_{2} \leqslant-1$ в любом угле Штольца $W_{\eta}$ с верииной в $e^{i \theta}$, то е е не является точкой роста функиии $f^{\prime}$.

ДоКАЗАТЕЛЬСтво неравенства $\delta_{1}+1 \leqslant \delta_{\eta}\left(f^{\prime}\right)$ не отличается от соответствующей части доказательства теоремы 5 .

Из определения $\delta_{2}$ получаем, что для $z=r e^{i \varphi} \in W_{\eta}$

$$
\limsup _{z \rightarrow e^{i \theta}} \operatorname{Re}\left\{\frac{f^{\prime \prime}\left(r e^{i \varphi}\right)}{f^{\prime}\left(r e^{i \varphi}\right)} e^{i \varphi}(1-r)\right\}=\delta_{2}+1
$$

Следовательно, для любого $\varepsilon>0$ сушествует $r_{\varepsilon} \in(0,1)$ такое, что при $z=r e^{i \varphi} \in W_{\eta}$, $r \geqslant r_{\varepsilon}$, выполняется неравенство

$$
\operatorname{Re}\left\{\frac{f^{\prime \prime}\left(r e^{i \varphi}\right)}{f^{\prime}\left(r e^{i \varphi}\right)} e^{i \varphi}\right\} \leqslant \frac{\delta_{2}+1+\varepsilon}{1-r} .
$$

Интегрируя это неравенство по отрезку $\left[r_{\varepsilon}, r\right]$, получим

$$
\left|f^{\prime}\left(r e^{i \varphi}\right)\right|(1-r)^{\delta_{2}+1+\varepsilon} \leqslant\left|f^{\prime}\left(r_{\varepsilon} e^{i \varphi}\right)\right|\left(1-r_{\varepsilon}\right)^{\delta_{2}+1+\varepsilon} \leqslant \max _{|z|=r_{\varepsilon}}\left[\left|f^{\prime}(z)\right|(1-|z|)^{\delta_{2}+1+\varepsilon}\right]
$$

т.е. $\left|f^{\prime}(z)\right|(1-|z|)^{\delta_{2}+1+\varepsilon}$ ограничено при $z \in W_{\eta},|z| \geqslant r_{\varepsilon}$. Из произвольности $\varepsilon>0$, как и в теореме 5 получаем неравенство $\delta_{\eta}\left(f^{\prime}\right) \leqslant \delta_{2}+1$.

Если $\delta_{2} \leqslant-1$, как и в теореме 5 приходим к выводу, что $e^{i \theta}$ не является точкой роста $f$.

Теорема $5^{\prime}$ доказана.

Таким образом, выражение $t(z, f)$, определяющее порядок ord $f$ локально однолистной функции $f$, играет важную роль при исследовании точек роста этой функции.

\section{СПИСОК ЦИТИРОВАННОЙ ЛИТЕРАТУРЫ}

[1] Pommerenke Ch. Linear-invariante Familien analytischer Funktionen. I // Math. Ann. 1964. V. 155. P. 108-154.

[2] Старков В. В. Теоремы регулярности для универсальных линейно-инвариантных семейств функций // Болгарский матем. ж. "СЕР ДИКА". 1985. Т. 11. С. 299-318.

[3] Харди Г. Расходящиеся ряды. М.: ИЛ, 1951.

(Я. Годуля) Институт математики Университета

Поступило

М. Кюри-Склодовской, г. Люблин, Польша

24.06.1999

(В.В. Старков) Петрозаводский государственный университет

E-mail: godula@golem.umcs.lublin.pl, 